\title{
Smoking and Methotrexate Inefficacy in Rheumatoid Arthritis: What About Underlying Molecular Mechanisms?
}
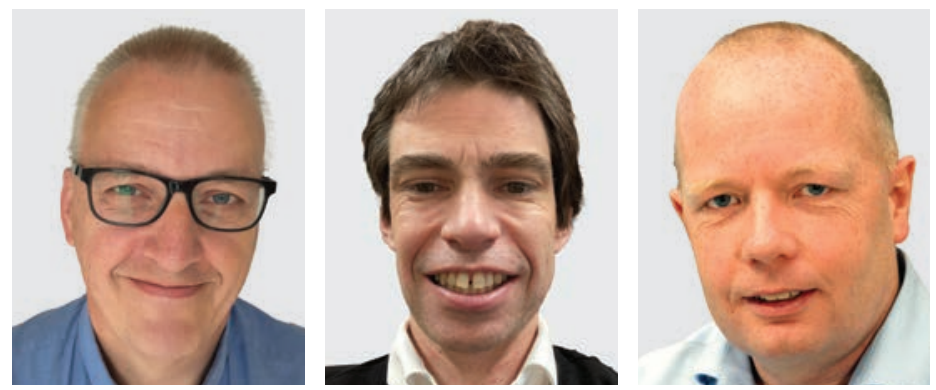

\author{
Gerrit Jansen $^{1}$, Maurits C.F.J. de Rotte ${ }^{2}$, and Robert de Jonge ${ }^{2}$
}

The study by Safy-Khan, et al in the current issue of The Journal of Rheumatology ${ }^{1}$ reports that in a methotrexate (MTX)-based treatment regimen for patients with early arthritis, current smoking was significantly associated with a smaller reduction of Disease Activity Score in 28 joints (DAS28) over time compared to noncurrent smoking. This negative effect of current smoking on DAS28 was dose-dependent: patients who smoked 10-19 cigarettes per day did worse than patients who smoked 1-9 cigarettes per day. Moreover, the effect was independent of concomitant prednisone use. These results support data from other clinical studies (reviewed in Ling, et $a l^{2}$ ) referring to smoking as a contributing factor to MTX nonresponse in patients with rheumatoid arthritis (RA). However, within these observations, questions related to underlying molecular mechanisms by which smoking adversely affects MTX efficacy have been largely underexposed. Here we will discuss some relevant factors emerging from MTX nonresponse prediction models from the perspective of the mechanism of action of MTX, drug resistance, and the role of smoking therein.

In brief, the mechanism of action of low-dose MTX treatment in RA involves MTX uptake in immune-competent cells, followed by its intracellular retention through conversion to polyanionic MTX-polyglutamate (MTX-PG) forms by the

${ }^{I}$ G. Jansen, PhD, Biochemist, Amsterdam Rheumatology and Immunology Center, Amsterdam University Medical Center, location VUmc;

${ }^{2}$ M.C. de Rotte, PhD, Clinical Chemist, $R$. de Jonge, PhD, Professor of

Clinical Chemistry, Department of Clinical Chemistry, Amsterdam

University Medical Center, Amsterdam, the Netherlands.

The authors declare no conflicts of interest relevant to this article.

Address correspondence to Dr. G. Jansen, Amsterdam Rheumatology and Immunology Center, Amsterdam University Medical Center, location VUmc, CCA - Rm 2.46, De Boelelaan 1117, 1081 HV Amsterdam, Amsterdam, the Netherlands.Email: g.jansen@amsterdamumc.nl. enzyme folylpolyglutamate synthetase. Polyglutamylation of MTX prevents MTX from being extruded from cells by selected members of the adenosine triphosphate (ATP)-binding cassette (ABC) drug efflux transporter family, including ABCC1-5 and ABCG2. Intracellularly, MTX-PGs inhibit several key enzymes in folate metabolism and purine biosynthesis de novo. These pharmacological inhibitions ultimately lead to the release of adenosine exerting antiinflammatory effects as well as inhibition of downstream signaling pathways driving inflammation. ${ }^{3}$ Polyglutamylation and efflux by $\mathrm{ABC}$ transporters are also critical processes in cellular homeostasis of natural folates, which are being hijacked by MTX.

Over the past decades, clinically directed laboratory studies on blood cells and synovial tissue identified multiple predictors for their association with MTX (in)efficacy in RA. These markers were based on the following: (1) altered pharmacogenetic/ genomic profiles of folate metabolism-related genes; (2) folate status-related altered epigenetics; (3) inflammation-related altered proteomic and metabolic profiles; and (4) altered serological and/or altered immunological marker profiles between MTX responders and nonresponders. ${ }^{2}$ Combinations of clinical and lifestyle variables, and selected laboratory variables determined at baseline, were then used to construct and validate models to predict MTX nonresponse after 3-12 months. ${ }^{45,6,7}$ Studies by Teitsma, et al for the U-Act-Early study utilized MTX response after 1-year treatment as an outcome measure and defined baseline DAS28 as the major contributor in the prediction model, along with current smoking and alcohol consumption. Inclusion of these variables revealed an area under the receiver-operating characteristic curve (AUC) of 0.75 for the U-Act-Early as development cohort and 0.68 for a validation cohort. ${ }^{4}$ De Rotte, et al included data from 2 clinical studies, MTX-R and tREACH, to predict insufficient response to 3-month MTX treatment

See Current smoking effect in CAMERA-II, page 1504 
in patients with RA before disease-modifying antirheumatic drug (DMARD) initiation. ${ }^{6}$ In their prediction model, baseline DAS28 (> 5.1) was again a contributor. Other variables such as the Health Assessment Questionnaire (HAQ; > 0.6), current smoking, BMI $\left(>25 \mathrm{~kg} / \mathrm{m}^{2}\right)$, single-nucleotide polymorphisms for $\mathrm{ABC}$ transporters $\mathrm{ABCB} 1$ (rs 1045642) and ABCC3 (rs4793665), and erythrocyte folate were additional variables contributing to the model that had an AUC of 0.80 for both the derivation and validation cohorts. Notably, within this model, current smoking had the highest relative contribution of the variables to the prediction scores. This model was recently externally validated for the U-Act-Early study, revealing an AUC of 0.75 and 0.71 for MTX nonresponse after 3 and 6 months, respectively. ${ }^{7}$ Interestingly, Sergeant, et al constructed an MTX nonresponse prediction model for European Alliance of Associations for Rheumatology response after 6 months, with an AUC of 0.74 that included disease activity measures (HAQ, tender joint count [TJC], DAS), and laboratory (rheumatoid factor $[\mathrm{RF}]$ ) and psychosocial (anxiety/depression) variables. ${ }^{5}$ Interestingly, current smoking was positively associated with MTX nonresponse in the univariate analysis. Together, lifestyle variables (BMI, smoking, alcohol), demographic factors (age, sex), disease activity measures (DAS, HAQ, TJC), and to a lesser extent, laboratory variables (erythrocyte folate, C-reactive protein/erythrocyte sedimentation rate, $\mathrm{RF}$ ), are the major contributors to MTX nonresponse. The future importance is dependent on the type of prediction model used, such as logistic regression or more complex machine learning models. ${ }^{8}$ Current smoking, lifestyle variables, and demographic factors have the advantage over laboratory variables of being relatively easy to implement in clinical practice.

Tobacco and (e-)cigarette smoke contain thousands of individual constituents, including many potentially toxic and carcinogenic components. ${ }^{9}$ When released in airway tissues and circulation, natural defense systems will be mobilized to minimize the harmful effects in the short and long run. ${ }^{10}$ Nevertheless, smoking is associated with extraarticular manifestations contributing to cardiovascular and respiratory comorbidities in patients with RA by imposing oxidative stress to endothelial cells, airway epithelium, and residing immune cells (e.g., macrophages). ${ }^{11,12}$ One prominent cellular defense system to toxic compounds is formed by the protein family of $\mathrm{ABC}$ drug efflux transporters. ${ }^{10,13}$ This family of 49 members with functional redundancy covers a broad substrate specificity of expelling toxic compounds as well as therapeutic drugs varying in chemical structure and charge. The prototypical ABC transporter P-glycoprotein (P-gp; ABCB1) has hydrophobic compounds as preferred substrates, whereas ABCC1-5 and ABCG2 preferentially export amphiphilic/ charged compounds/drugs. To this end, DMARDs are also among the substrates of $A B C$ transporters, with $A B C B 1$ capable of exporting glucocorticoids (prednisone) and $\mathrm{ABCC} 1-5$ and ABCG2 capable of extruding MTX, hydroxychloroquine, sulfasalazine, and leflunomide. ${ }^{13,14}$ Thus, upregulation of $\mathrm{ABC}$ transporters can contribute to conferring diminished drug response. ${ }^{14}$ An important aspect of $\mathrm{ABC}$ transporters is that beyond their pharmacological function, they also harbor a phys- iological function by exporting substrates related to immune response (e.g., prostaglandins, leukotrienes, and sphingolipids) to facilitate the migration of immune cells to lymph nodes. ${ }^{13}$ In fact, in RA, expression of selected $A B C$ transporters on peripheral blood lymphocytes and synovial macrophages has been associated with RA disease activity (role for P-gp) and diminished response to DMARDs, including MTX (through ABCC1-5 and ABCG2) and prednisone (through P-gp). 13,15,16,17. Moreover, $\mathrm{ABCC} 1-5$ and $\mathrm{ABCG} 2$ play a role in folate homeostasis, ${ }^{14}$ which indirectly affects MTX polyglutamylation and efficacy. For these reasons, selected $\mathrm{ABC}$ transporters (wildtype variant) and erythrocyte folate were incorporated as variables in MTX nonresponse prediction models. ${ }^{6}$ Of note, a low baseline erythrocyte folate content has been associated with MTX nonresponsiveness after 3 months of therapy. ${ }^{18}$ From this perspective, smokers were also identified by significantly lower erythrocyte folate levels than nonsmokers. ${ }^{19}$ There is no particular evidence that concentrations of smoke components may inhibit intestinal and cellular folate transporters; rather, lower blood folate concentrations may be due to lifestyle-related lower folate intake of fruits and vegetables by smokers. ${ }^{20}$ Smoking has also been associated with lower accumulation of long-chain MTX-PGs in erythrocytes of patients with RA during long-term MTX treatment. ${ }^{21}$ In prospective clinical studies, erythrocyte accumulation of these long chain MTX-PGs over 3-9 months was shown to correlate with a better reduction of DAS28 scores in both patients with RA and those with juvenile idiopathic arthritis, although large interpatient variabilities in MTX-PG concentrations were observed. ${ }^{22,23}$ Acknowledging that assessments of erythrocyte folate concentrations and MTX-PG levels serve as a bona fide marker for MTX response and MTX nonresponse prediction models, it has to be realized that erythrocytes are not the primary blood cells involved in RA disease pathogenesis. Also, mature erythrocytes have no regulated folate metabolism such as in peripheral blood mononuclear cells. As such, future directions for MTX therapeutic drug monitoring and MTX-PG analyses warrant extension to relevant immune cells, which are analytically feasible by combined liquid chromatography and mass spectrometry technology with labeled internal standards. ${ }^{24}$ Also, inclusion of a laboratory marker to better define the extent and duration of smoking, such as cotinine, ${ }^{25}$ may be helpful for correlations with clinical response and laboratory variables. These studies may guide further improvement of current MTX nonresponse prediction models by inclusion of novel variables.

The results in the study by Safy-Khan, et al ${ }^{1}$ show that the adverse effect of current smoking on MTX treatment efficacy for patients with early arthritis was independent of concomitant prednisone use; therefore, it suggests no apparent drug interaction between MTX and prednisone. The distinct mechanism of action of both drugs, and the notion that prednisone has no involvement with $\mathrm{ABC}$ transporters being relevant for folate/ MTX metabolism, is consistent with this clinical observation.

\section{REFERENCES}

1. Safy-Khan M, de Hair MJ, Welsing PMJ, van Laar JM, Jacobs JW; Society for Rheumatology Research Utrecht. Current smoking 
negatively affects the response to methotrexate in RA in a dose-responsive way, independent of concomitant prednisone use. J Rheumatol 2021;48:1504-7.

2. Ling S, Bluett J, Barton A. Prediction of response to methotrexate in rheumatoid arthritis. Expert Rev Clin Immunol 2018;14:419-29.

3. Brown PM, Pratt AG, Isaacs JD. Mechanism of action of methotrexate in rheumatoid arthritis, and the search for biomarkers. Nat Rev Rheumatol 2016;12:731-42.

4. Teitsma XM, Jacobs JW, Welsing PM, de Jong PH, Hazes JM, Weel AE, et al. Inadequate response to treat-to-target methotrexate therapy in patients with new-onset rheumatoid arthritis: development and validation of clinical predictors. Ann Rheum Dis 2018;77:1261-7.

5. Sergeant JC, Hyrich KL, Anderson J, Kopec-Harding K, Hope HF, Symmons DP, et al. Prediction of primary non-response to methotrexate therapy using demographic, clinical and psychosocial variables: results from the UK Rheumatoid Arthritis Medication Study (RAMS). Arthritis Res Ther 2018;20:147.

6. de Rotte MC, Pluijm SMF, de Jong PH, Bulatovic CM, Wulffraat NM, Weel AE, et al. Development and validation of a prognostic multivariable model to predict insufficient clinical response to methotrexate in rheumatoid arthritis. PLoS One 2018;13:e0208534.

7. Gosselt HR, Verhoeven MM, de Rotte MC, Pluijm SM, Muller IB, Jansen G, et al. Validation of a prognostic multivariable prediction model for insufficient clinical response to methotrexate in early rheumatoid arthritis and its clinical application in Evidencio. Rheumatol Ther 2020;7:837-50.

8. Gosselt HR, Verhoeven MM, Bulatovic-Calasan M, Welsing PM, de Rotte MC, Hazes JM, et al. Complex machine-learning algorithms and multivariable logistic regression on par in the prediction of insufficient clinical response to methotrexate in rheumatoid arthritis. J Pers Med 2021;11:44.

9. Stedman RL. The chemical composition of tobacco and tobacco smoke. Chem Rev 1968;68:153-207.

10. Kaur G, Gaurav A, Lamb T, Perkins M, Muthumalage T, Rahman I. Current perspectives on characteristics, compositions, and toxicological effects of e-cigarettes containing tobacco and menthol/ mint flavors. Front Physiol 2020;11:613948.

11. Chang K, Yang SM, Kim SH, Han KH, Park SJ, Shin JI. Smoking and rheumatoid arthritis. Int J Mol Sci 2014;15:22279-95.

12. Aridgides DS, Mellinger DL, Armstrong DA, Hazlett HF, Dessaint JA, Hampton TH, et al. Functional and metabolic impairment in cigarette smoke-exposed macrophages is tied to oxidative stress. Sci Rep 2019;9:9624.

13. van de Ven R, Oerlemans R, Van der Heijden JW, Scheffer GL, de Gruijl TD, Jansen G, et al. ABC drug transporters and immunity: novel therapeutic targets in autoimmunity and cancer. J Leukoc Biol 2009;86:1075-87.

14. Van der Heijden JW, Dijkmans BA, Scheper RJ, Jansen G. Drug insight: resistance to methotrexate and other disease-modifying antirheumatic drugs-from bench to bedside. Nat Clin Pract Rheumatol 2007;3:26-34.
15. Oerlemans R, Blits M, Dijkmans BAC, Van der Heijden JW, Lems WF, Scheffer GL, et al. Expression profiling of $A B C$ transporters in peripheral blood lymphocytes and monocyte-derived macrophages of rheumatoid arthritis patients. J Mol Clin Med 2020;3:47-60.

16. Van der Heijden JW, Oerlemans R, Tak PP, Assaraf YG, Kraan MC, Scheffer GL, et al. Involvement of breast cancer resistance protein expression on rheumatoid arthritis synovial tissue macrophages in resistance to methotrexate and leflunomide. Arthritis Rheum 2009;60:669-77.

17. Tsujimura S, Saito K, Nawata M, Nakayamada S, Tanaka Y. Overcoming drug resistance induced by $\mathrm{P}$-glycoprotein on lymphocytes in patients with refractory rheumatoid arthritis. Ann Rheum Dis 2008;67:380-8.

18. de Rotte MC, de Jong PH, Pluijm SM, Calasan MB, Barendregt PJ, van Zeben D, et al. Association of low baseline levels of erythrocyte folate with treatment nonresponse at three months in rheumatoid arthritis patients receiving methotrexate. Arthritis Rheum 2013;65:2803-13.

19. Pfeiffer CM, Sternberg MR, Schleicher RL, Rybak ME. Dietary supplement use and smoking are important correlates of biomarkers of water-soluble vitamin status after adjusting for sociodemographic and lifestyle variables in a representative sample of U.S. adults. J Nutr 2013; 143:957S-65S.

20. Okumura K, Tsukamoto H. Folate in smokers. Clin Chim Acta 2011;412:521-6.

21. Stamp LK, O'Donnell JL, Chapman PT, Zhang M, Frampton C, James J, et al. Determinants of red blood cell methotrexate polyglutamate concentrations in rheumatoid arthritis patients receiving long-term methotrexate treatment. Arthritis Rheum 2009;60:2248-56.

22. de Rotte MC, den Boer E, de Jong PH, Pluijm SM, Calasan MB, Weel AE, et al. Methotrexate polyglutamates in erythrocytes are associated with lower disease activity in patients with rheumatoid arthritis. Ann Rheum Dis 2015;74:408-14.

23. Ćalasan MB, den Boer E, de Rotte MC, Vastert SJ, Kamphuis S, de Jonge R, et al. Methotrexate polyglutamates in erythrocytes are associated with lower disease activity in juvenile idiopathic arthritis patients. Ann Rheum Dis 2015;74:402-7.

24. Muller IB, Hebing RCF, Jansen G, Nurmohamed MT, Lems WF, Peters GJ, et al. Personalized medicine in rheumatoid arthritis: methotrexate polyglutamylation revisited. Expert Rev Prec Med Drug Dev 2018;3:331-4.

25. Maska LB, Sayles HR, O’Dell JR, Curtis JR, Bridges SL, Jr., Moreland LW, et al. Serum cotinine as a biomarker of tobacco exposure and the association with treatment response in early rheumatoid arthritis. Arthritis Care Res 2012;64:1804-10. 